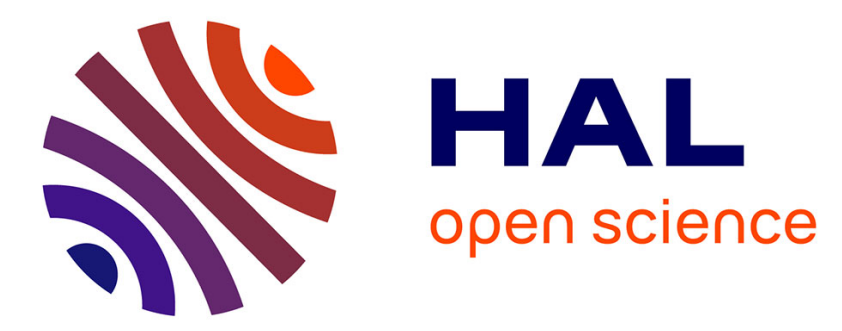

\title{
Oxygen supply of the heart and electrocardiogram potentials with reversed polarity in sleeping and resting honey bees
}

Walter Kaiser, Theo Weber, Dietmar Otto, Anton Miroschnikow

\section{To cite this version:}

Walter Kaiser, Theo Weber, Dietmar Otto, Anton Miroschnikow. Oxygen supply of the heart and electrocardiogram potentials with reversed polarity in sleeping and resting honey bees. Apidologie, 2013, 45 (1), pp.73-87. 10.1007/s13592-013-0229-2 . hal-01234706

\author{
HAL Id: hal-01234706 \\ https://hal.science/hal-01234706
}

Submitted on 27 Nov 2015

HAL is a multi-disciplinary open access archive for the deposit and dissemination of scientific research documents, whether they are published or not. The documents may come from teaching and research institutions in France or abroad, or from public or private research centers.
L'archive ouverte pluridisciplinaire HAL, est destinée au dépôt et à la diffusion de documents scientifiques de niveau recherche, publiés ou non, émanant des établissements d'enseignement et de recherche français ou étrangers, des laboratoires publics ou privés. 


\title{
Oxygen supply of the heart and electrocardiogram potentials with reversed polarity in sleeping and resting honey bees
}

\author{
Walter KaISER $^{1,4}$, Theo WeBer $^{2}$, Dietmar OtTo ${ }^{2}$, Anton MiroschniKow ${ }^{3}$ \\ ${ }^{1}$ Institut für Zoologie der Technischen Universität Darmstadt, Schnittspahnstr. 3, D-64287 Darmstadt, Germany \\ ${ }^{2}$ Max-Planck-Institut für Ornithologie, D-82319, Seewiesen, Germany \\ ${ }^{3}$ Laboratory of Molecular Brain Physiology and Behavior Development, Genetics and Molecular Physiology Unit, \\ LIMES, Universität Bonn, Carl-Troll-Str. 31, D-53115 Bonn, Germany \\ ${ }^{4}$ Godesberger Hof 14, D-53173 Bonn, Germany
}

Received 7 November 2012 - Revised 10 May 2013 - Accepted 15 July 2013

\begin{abstract}
A novel active ventilatory mechanism of the dorsal air sacs in the abdomen of forager honey bees is described. These air sacs supply the heart with oxygen. During sleep and rest, the air sacs undergo frequent volume changes. The dilation of the air sacs is caused by the activity of hitherto unidentified muscles. Their subsequent collapse is probably due to elastic components in the walls of the sacs. The volume changes also occur when, during discontinuous resting ventilation, ventilatory movements of the abdomen are absent. They are always combined with an increase in heart rate. The heart thus receives oxygen when it needs it and can therefore function effectively during sleep, to supply the brain with nutrients necessary for energy-demanding sleep-specific functions. During sleep, rest, and induction of chill coma, periodic heartbeat reversals were also discovered. The biological significance of these heartbeat reversals remains to be elucidated.
\end{abstract}

honey bee / sleep / heart function / oxygen supply / electrocardiogram

\section{INTRODUCTION}

The existence of a sleep-like state sharing many characteristics with mammalian sleep is well established in honey bees (see Eban-

Electronic supplementary material The online version of this article (doi:10.1007/s13592-013-0229-2) contains supplementary material, which is available to authorized users.

Corresponding author: W. Kaiser, wkaiser@uni-bonn.de

This paper is dedicated to Jana Steiner-Kaiser and Theo Zaschka. They both gave extremely valuable long-term support to one of us (W.K.) - with translations and constructive criticism and the supply of superb custom-built apparatuses, respectively.

Manuscript editor: Bernd Grünewald
Rothschild and Bloch 2012; Klein and Seeley 2011; Klein et al. 2010 for a recent summary of the relevant literature). Sleep in humans and other mammals is not a passive state but rather an active, energy-demanding process (history: Dement 2011; learning and memory: Peigneux and Smith 2011). That this also applies to the honey bee first became apparent through the discovery of repeated bouts of antennal movements during nightly sleep in forager honey bees (Kaiser 1988; Sauer et al. 2003; see Figure 1). The solitary bees studied so far differ in this respect from the honey bee (Kaiser 1995): Some species show antennal immobility lasting for hours during rest at night. A first insight into the significance of brain functions during sleep in the honey bee comes from the papers of Beyaert et al. (2012) studying mem- 


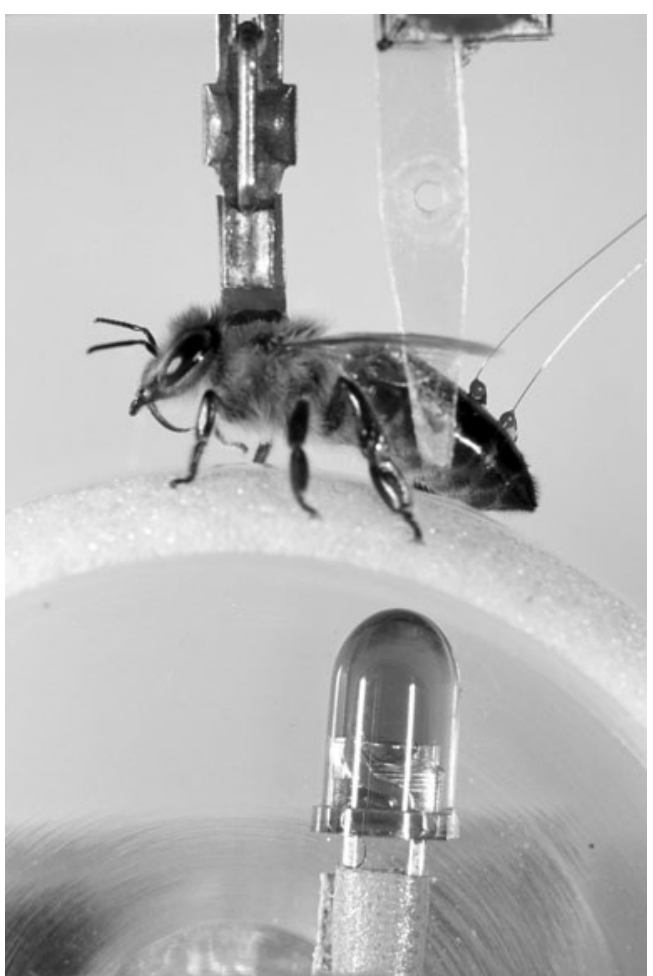

Figure 1. Worker honey bee walking (note raised head and antennae) on a treadwheel. Two wire electrodes were fixed to the abdominal tergites 5 and 6. A diode emitting infrared light $(950 \mathrm{~nm})$ was mounted inside the treadwheel. The infrared light could pass through the transparent wheel and then enter the bee's abdomen. An infrared-sensitive video camera (not shown) was mounted above the abdomen. Because the abdominal cuticle of honey bees is transparent for infrared light, the camera could visualize internal organs of the abdomen (see Figure 2 and also the Videos S1 and S2 in the digital supplementary material)

ory consolidation and of Klein et al. (2010) studying the precision of communication through the waggle dance in these animals.

Metabolic processes are temperature-dependent. Because forager honey bees interrupt the production of heat with their thoracic musculature during sleep in the hive (Kaiser 1988), they choose sleep sites which are warm enough to allow metabolic processes to occur $\left(25-29{ }^{\circ} \mathrm{C}\right.$ : Kaiser et al. 2002;
Schmolz et al. 2002). Nevertheless, some energy conservation during sleep is possible, since the brood area, which is kept at around $35{ }^{\circ} \mathrm{C}$ by younger bees, is avoided as a sleeping site.

Metabolic processes in the brain of the honey bee depend on the supply of nutrients contained in the blood (hemolymph) reaching the brain only when the heart is engaged in pumping movements. The brain then is bathed by the blood exiting the anterior opening of the "aorta." This thin-walled tube is the anterior continuation of the thick-walled muscular tube of the abdominal heart. The hemolymph receives its nutrients by bathing the abdominal organs. The blood then enters the dorsally located tubular heart via small openings (ostia) and is then pumped to the head (open circulatory system; Snodgrass 1956).

The goal of this study was, therefore, to investigate the activity and function of the honey bee's heart during sleep and rest. We used a technique that was successful in previous experiments: We mounted the bees above a lightweight treadwheel (Sauer et al. 2003, 2004). This approach not only enabled us to obtain electrophysiological recordings from animals which could walk and move their antennae, head, and abdomen but also made it possible to simultaneously transilluminate the abdomen with infrared light and visualize interior organs with video techniques.

In this paper, we present the results of longlasting experiments (days) in which the vitality of the experimental animals and the stability of the electrophysiological recordings became apparent.

\section{MATERIALS AND METHODS}

\subsection{Transillumination of the abdomen without electrophysiological recording}

\subsubsection{Experimental animals}

We used only young, pollen-foraging honey bees (Apis mellifera) with intact fur. They were captured on their return to the hive. At the start of each experiment, we placed around 20 bees in a holding 
cage in which high humidity, and a temperature of approximately $25{ }^{\circ} \mathrm{C}$ prevailed. The cage was illuminated by two fluorescent tubes operating on high-frequency alternating current $(24 \mathrm{kHz})$, one providing "daylight" (Osram, DS/E 9/21) and one providing UV-A (Osram, DS/E 9/78). The illumination was switched on at sunrise and off at sunset. In the cage, the animals were fed with undiluted acacia honey ad libitum. During the experiments, the bees were offered $2 \mathrm{M}$ sugar water in order to minimize the amount of waste-products in the gut which could reduce the quality of the transillumination picture. Several bees were lightly anaesthetized by cooling and a small plastic tab $(2 \times 4 \mathrm{~mm})$ was glued vertically on the thorax of each animal with wax-resin (1:9). The fur of the abdominal tergites 4-6 was removed by using adhesive tape attached to the tips of fine tweezers. After this treatment, the animals behaved normally (e.g., they showed social behavior) in the holding cage. On the following day, one of these bees was placed in the experimental apparatus.

Two intact animals were studied using transillumination without electrophysiology. (In addition, four animals were studied combining transillumination and electrophysiology; see Section 2.2.1).

The experiments are characterized by the following parameters: (a) duration of the experiment; (b) duration of the continuous video recording (transillumination plus head/antennae) containing the first dark phase (each dark phase was a period of darkness imposed upon a honey bee designed to roughly match nighttime); (c) duration of the dark phase; (d) duration of the continuous video recording during the "chill coma experiment" (see Section 2.1.3) in the afternoon after the first night; (e) duration of the continuous video recording containing the second dark phase. Animal 1: (a) 1 day 22 h 5 min (10-12 September 2002); (b) 12 h $18 \mathrm{~min}$; (c) 10 h 45 min; (d) 3 h 4 min; (e) 15 h 10 min; Animal 2, (a) 1 day 8 h 55 min (20-21 September 2002); (b) 15 h 4 min; (c) 11 h 30 min; (d) 3 h 4 min.

\subsubsection{Experimental apparatus}

During the entire experiment, each bee was fixed above a treadwheel via the small plastic tab on its thorax (Figure 1 and Figure S1 in the supplementary material; in this experimental series, no recording wires were attached to the animal). The bee's legs had firm contact with the wheel, which could be easily rotated. The animal could thus engage in locomotor activity and make extensive abdominal movements as well as head and antennal movements. Only larger lateral abdominal movements were restricted by two small plastic plates located to the right and left of the abdomen. These plates prevented the wings from covering the abdomen and thus allowed the dorsally mounted video camera to obtain a clear image of the transilluminated abdomen.

The treadwheel was made of Lucite and had a diameter of $50 \mathrm{~mm}$ and a width of $13 \mathrm{~mm}$. The two edges of the wheel were covered with a rough plastic surface (Röhm, Rohacell) which provided grip for the tarsi of the animals. Between the edges, the Lucite was clear, in order to allow the passage of infrared light $(950 \mathrm{~nm})$ provided by one infrared light emitting diode (Siemens, LED 274-3), mounted inside the wheel, which transilluminated the abdomen.

In order to prevent the treadwheel from becoming contaminated by sugar water offered from a cannula (see below), the bee was only fed by hand, during the day, using a fine brush offering droplets of $2 \mathrm{M}$ sugar water.

Throughout each experiment, the behavior of the bee was recorded continuously by two infraredsensitive video cameras that were connected to time lapse recorders (Panasonic, AG-6720). One camera (Panasonic, WV-CD 22) was mounted laterally and provided an image of the head and thorax seen in silhouette (lighting conditions: four infrared-emitting diodes, Siemens, LED 271, transilluminated an opaque glass screen mounted behind the animal). The second camera (Panasonic, WV-BP 554) was mounted above the animal and viewed the transilluminated abdomen (light source: one LED in the treadwheel; see above). The sensitivity of both video cameras for light emitted by the fluorescent tubes that illuminated the animal during the day was suppressed with cutoff filters (Schott, RG 830) mounted on the objectives.

The entire experimental apparatus was located in a light-tight cage within which an ambient temperature of $25.3-25.6{ }^{\circ} \mathrm{C}$ was maintained by a radiator connected to an ultrathermostat. Illumination inside the cage was provided by lighting identical to that used in the holding cage. The lighting was turned on and off at the local times of sunrise and sunset, 
respectively. Only one experimental animal at a time could be studied with this experimental apparatus.

\subsubsection{Chill coma experiments}

Chill coma experiments were carried out in order to compare physiological events during sleep and rest with those occurring during chill coma. The entire experimental apparatus, with the animal, was slowly cooled to $5.8-5.9{ }^{\circ} \mathrm{C}$ and maintained at this temperature for approximately $1 \mathrm{~h}$. Subsequently, the bee and the apparatus were slowly re-warmed to the starting temperature (see above).

\subsubsection{Data evaluation}

All video tapes containing the information about date and time of day as well as the number of video half-frames were played back at recording speed, and detailed notes were taken. The tapes of the first two nights of the two animals (which were recorded with full time resolution, $3 \mathrm{~h}$ mode) then were played back, again, and the times of occurrence of specific events were transferred to a computer: A key of the keyboard was pressed briefly when the air sac under consideration was expanded. Another key was pressed when, during discontinuous resting ventilation, ventilatory abdominal length changes started, and it was released only when the burst of ventilatory movements had ended. Thus, the duration of the relevant ventilatory burst could be stored. Differences in the degree and duration of air sac expansions were neglected.

\subsection{Experiments combining transillumination and electrophysiology}

\subsubsection{Experimental animals}

Four animals were studied. In one animal, the wings had been trimmed; in the other three animals the wings remained intact (see Figure 1). We did not see differences in the results and the behavior related to the different treatments. The animals were fed by hand with $2 \mathrm{M}$ sugar water (see Section 2.1.2). Chill coma experiments were performed on three of the four animals (see Section 2.1.3).
The experiments are characterized by the following parameters: (a) duration of the experiment; (b) duration of continuous video recording (transillumination plus head/antennae) containing the first dark phase; (c) duration of continuous recording of electrical signals (analog tape recorder) containing the first dark phase; (d) duration of dark phase; (e) duration of continuous video recording containing the chill coma experiment; (f) duration of continuous recording of electrical signals containing the chill coma experiment; (g) duration of video recording containing the second nightly dark phase; (h) duration of recording of electrical signals containing the second nightly dark phase.

Animal 1 (wings clipped): (a) 3 days 22 h 35 min (15-19 August 2000); (b) $12 \mathrm{~h} 15 \mathrm{~min}$; (c) $12 \mathrm{~h}$ $19 \mathrm{~min}$; (d) 9 h $30 \mathrm{~min}$; (e) $5 \mathrm{~h} 14 \mathrm{~min}$; (f) $3 \mathrm{~h}$ 49 min; (g) 15 h 10 min; (h) 15 h 51 min. Animal 2 (wings intact): (a) 1 day 17 h 47 min (1-3 September 2000); (b) 15 h $19 \mathrm{~min}$; (c) $10 \mathrm{~h} 12 \mathrm{~min}$; (d) $10 \mathrm{~h}$ 30 min; (e) 5 h 54 min (chill coma experiment, 2 h $29 \mathrm{~min}$ ); (f) 5 h $59 \mathrm{~min}$. Animal 3 (wings intact): (a) 21 h 28 min (15-16 September 2000); (b) 14 h 9 min; (c) $14 \mathrm{~h} 1 \mathrm{~min}$; (d) $13 \mathrm{~h}$. Animal 4 (wings intact): (a) 2 days 7 h 52 min (17-19 October 2000); (b) 15 h 8 min; (c) 16 h 25 min; (d) 13 h; (e) 5 h 9 min (chill coma experiment, $3 \mathrm{~h} 49 \mathrm{~min}$ ); (f) $4 \mathrm{~h} 6 \mathrm{~min}$; (g) $15 \mathrm{~h}$ $15 \mathrm{~min}$; (h) 8 h $13 \mathrm{~min}$.

\subsubsection{Electrophysiology}

The electrodes were made of insulated copper wire with a diameter of $56 \mu \mathrm{m}$. Copper wire was used since it is more flexible than steel wire and thus did not transmit mechanical forces to the bee's abdomen. In addition, copper wire is not toxic for insects when used in myogram recordings. In our case, we did not notice any deterioration of the recording during the experiments lasting several days.

A drop of dental wax was applied close to the ends of the electrodes. Under a microscope, the tips were cut off so that they extended approximately $0.16 \mathrm{~mm}(0.24 \mathrm{~mm}$ in Section 3.3) beyond the drop of wax. The free ends of the wires were inserted through small holes drilled into the tergites of the anesthetized bee's fifth and sixth abdominal segments, close to the right side of the heart. The drops of wax acted as stoppers. The freshly cut surface of 
the wire tips made electrical contact with the hemolymph of the pericardial sinus of the bee's abdomen and thus could pick up electrical signals produced by the heart (electrocardiogram (ECG)) and other muscles located in this sinus. Together with the wax stoppers, the recording electrodes were fastened to each tergite with a drop of wax-resin. The holes had been drilled under microscopic control, using brief transillumination of the abdomen with highintensity cold light. They were located in the bristlefree rostral parts of the tergites, so that neither the heart nor the neighboring air sacs were damaged (see Snodgrass 1956 for anatomical details). The electrode in the fifth abdominal segment was connected to the positive input of the amplifier, and the electrode in the sixth segment (where the end of the cardiac tube is located) was connected to the negative input (bipolar recording).

We used a differential amplifier (WPI Electronics, DAM-5A). This was connected to a band pass filter (Krohn-Hite, 3700) whose filter range was $2 \mathrm{~Hz}-3 \mathrm{kHz}$. The output of this electronic filter was connected to a loudspeaker and also to an analog tape recorder (Racal Electronics, Store 14 DS). At a tape speed of 15/16 ips, the extreme frequency values for the FM-channels of this tape recorder were 0 and $313 \mathrm{~Hz}$ and at $17 / 8$ ips 0 and $626 \mathrm{~Hz}$, respectively. One of the DR-channels was used to record clock time, so that, during data analysis, we could make exact comparisons between the electrophysiological events and the simultaneous overt behavior of the experimental bee recorded on video tape together with the time information. During two experiments, a microphone that was connected to the video recorder recording the transillumination of the abdomen was positioned in front of the loudspeaker. Thus, the occurrence of the sound produced by the electrophysiological signals could be recorded in perfect synchrony with the appearance of the physiological events on the video monitor. During the course of an experiment, comparisons were made of the events seen on the video monitor and on the oscilloscope screen with the simultaneous sound from the loudspeaker.

\subsubsection{Video recording}

In these experiments and those of Section 2.3, a further video camera (Philips, LDH 0703/30) was mounted lateral to the bee's abdomen and produced a highly magnified silhouette of the recording wires. This camera registered ventilatory movements (lengthening and shortening of the abdomen): The position of one of the wires on the video screen was scanned and measured continuously by a special custom-built video interface (Sauer et al. 2003); these measurements were led, as analog signals, to an FMchannel of the Racal tape recorder and registered simultaneously with the electrophysiological signals.

\subsubsection{Data analysis}

The data recorded with the Racal tape recorder-electrophysiological signals and abdominal movements - were later visualized on a fast fourchannel pen recorder (Gould Electronics, 2400 S; frequency response $0-50 \mathrm{~Hz}$ at full-scale deflection and $0-125 \mathrm{~Hz}$ at one fifth full-scale deflection). During data transfer to the paper chart, the tape machine ran at the original recording speed. The data on paper were evaluated by detailed visual inspection and related to the simultaneous behavior of the animals and to the state of their internal organs seen in transillumination and registered with video tapes. Relevant sections of the paper recordings were scanned into a computer for further evaluation and graphical representation.

\subsection{Electrophysiology and behavior without transillumination}

\subsubsection{Experimental animals}

The first three bees (nos. 1-3) were engaged in long-term experiments during which we did not want to disturb the animals by feeding them by hand. Instead, they were fed continuously from a cannula connected to a vessel filled with diluted acacia honey. In these three bees, the front legs had to be removed to prevent them from touching the tip of the cannula and thereby contaminating the treadwheel. The wings were also trimmed because they could touch the recording wires and thereby produce electrical disturbances. These operations were carried out directly after the bees had been caught. The animals were then transferred to the holding cage where they were active and had social contact with the intact control bees. 
In bees nos. 4 and 5, the front legs were left intact. Food (diluted acacia honey) was offered at certain times only by moving the cannula by hand toward the animal until its proboscis could reach it. Only the wings were trimmed, a procedure that, later, could also be avoided (see Figure 1 and Section 2.2.1). Nevertheless, the results obtained from all five bees did not differ from those of the intact bees (see Sections 2.2.1 and 3.2).

The experiments are characterized by the following parameters: (a) duration of the experiment; (b) total duration of the continuous video recording (head/antennae) containing the dark phase(s); (c) total duration of the continuous recording of the electrical signals with analog tape recorder containing the dark phase(s); (d) duration of dark phase.

Animal 1: (a) 4 days 18 h 30 min (24-29 June 1995); (b) 3 days 17 min containing three dark phases; (c) 1 days $7 \mathrm{~h} 16$ min containing two dark phases; (d) $7 \mathrm{~h}$ 56 min. Animal 2: (a) 5 days 35 min (8-13 July 1995); (b) 3 days $13 \mathrm{~h} 19$ min containing four dark phases; (c) 3 days 2 h 48 min containing five dark phases; (d) $8 \mathrm{~h}$ 7 min. Animal 3: (a) 11 days 18 h 40 min (14-26 September 1995); (b) 10 days $1 \mathrm{~h} 37$ min containing ten dark phases; (c) 5 days 8 h 34 min containing seven dark phases; (d) $11 \mathrm{~h} 8 \mathrm{~min}$. Animal 4 (front legs intact, wings clipped): (a) 1 day 23 h 23 min (5-7 October 1995); (b) 1 day $18 \mathrm{~h} 7$ min containing two dark phases; (c) 1 day 7 h 50 min containing two dark phases; (d) $12 \mathrm{~h} 21 \mathrm{~min}$. The figures shown in Section 3.3 are derived from the experiment with this animal. Animal 5 (front legs intact, wings clipped): (a) 2 days 15 h $57 \mathrm{~min}$ (18-21 October 1995); (b) 2 days $12 \mathrm{~h} 31 \mathrm{~min}$ containing three dark phases; (c) 1 day 21 h $53 \mathrm{~min}$ containing three dark phases; (d) 13 h 3 min.

\section{RESULTS}

\subsection{Transillumination of the abdomen without electrophysiological recording}

\subsubsection{General findings}

The basic result of this section is the following: One can visualize interior abdominal organs of bees using a single diode emitting short-wavelength infrared radiation combined with an infrared-sensitive video camera. The dorsal view of the abdomen reveals a clear picture of the medially located tubular heart and of the laterally located dorsal air sacs of the tracheal system that send fine branches to the heart (see Figures 1 and 2 which are taken from experiments described in Section 3.2, see also Figure S1, Videos S1, and S2 in the supplementary material).

\subsubsection{Repeated volume changes of the dorsal air sacs during discontinuous resting ventilation}

To our surprise, these air sacs undergo marked and repeated volume changes during the absence of abdominal ventilatory movements, i.e., when the animal is engaged in discontinuous resting ventilation. Figure 2 shows the left air sacs of the abdominal segments 5 and 6 (the right-hand side was obscured by the wax droplets fixing the electrophysiological recording wires to the abdominal tergites, since this figure is taken from the experiments described in Section 3.2). Figure 2a shows the air sacs in the collapsed state whereas, in Figure 2b, the end of the phase of the rapid dilation of the air sacs is shown. The collapse, which then follows, is a much slower process, compared with that of dilation (see also videos $\mathrm{S} 1$ and $\mathrm{S} 2$ in the supplementary material).

A careful inspection of the video image during the experiments and during the later replay of the recorded video tapes revealed two characteristic physiological events that were combined with the appearance of the air sac dilations during sleep and rest: (1) The occurrence of air sac dilations was always accompanied by an increase in heartbeat frequency (see Section 3.3 for quantitative data). When the heart showed phases of inactivity (which can last up to $1.1 \mathrm{~min}$ during nightly sleep: Kaiser et al. 1996), air sac dilations, occurring as single events or in a sequence of a few, were, in most cases, accompanied by a single heartbeat or several heartbeats. (2) A relationship also existed between the occurrence of bursts of ventilatory movements of the abdomen during discontinuous resting ventilation and the frequency of air sac dilations (number of dilations 

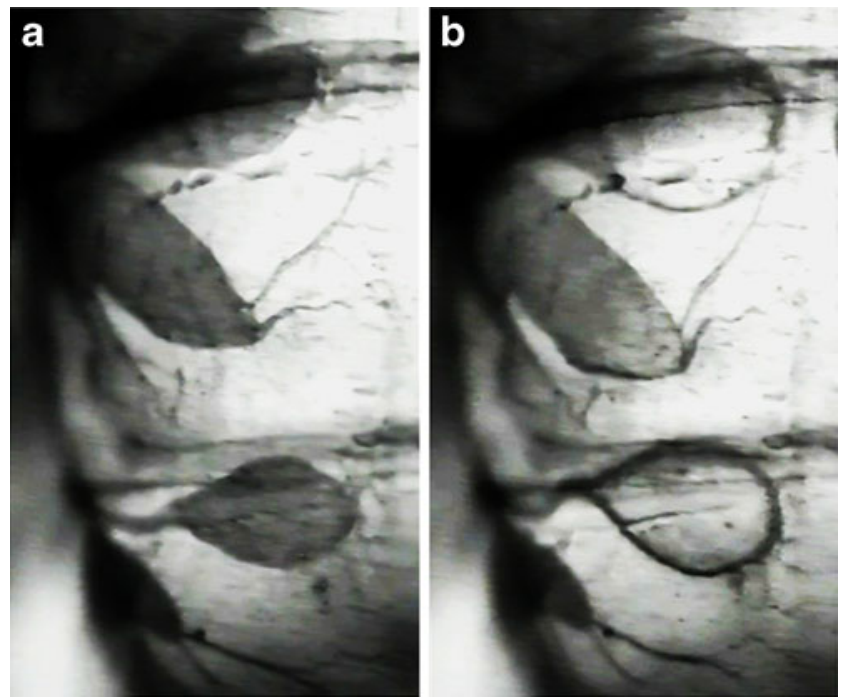

Figure 2. a, b Transillumination of the abdomen with infrared light (see Figure 1): dorsal views of the left halves of abdominal segments 5 and 6 . The right sides were obscured by the electrodes with their wax droplets. The tracheal structures (air sacs and their arborizations) reflect infrared light and thus appear dark in transillumination. The longitudinal structures seen on the right-hand edges of the pictures are the left borders of the tubular heart to which the dorsal air sacs send tracheal tubes with arborizations. Upper parts of the pictures: segment 5, lower parts: segment 6 . In each segment, two dorsal air sacs are present on each side of the heart. a Air sacs in the collapsed state, b dilated air sacs. The anterior air sacs show the most pronounced volume changes. The change in size of the posterior air sac of segment 5 is less pronounced. The posterior dorsal air sac of segment 6 was not observed to undergo volume changes in our experiments. See the Videos S1 and S2 in the supplementary material

per unit of time): During the burst, the frequency increased and, during the subsequent phase of ventilatory inactivity of the abdomen, the frequency of the dilations slowly declined to a plateau-like level. Figure 3 and its caption show the results of detailed analyses of these events during the first experimental nights of the two animals.

\subsubsection{Long-lasting dilations of the dorsal air sacs during active warming-up, rapid walking, and chill coma}

Phases of active warming-up, which precede phases of rapid walking on a treadwheel, occur after prolonged periods of rest (Kaiser 1988). In honey bees, the increase in body temperature is achieved by activation of the thoracic muscles, which is accompanied by rapid ventilatory pumping movements of the abdomen. The transillumination experiments communicated here revealed that, during such phases, the dorsal air sacs were constantly dilated but showed additional, repeated small dilations at a rhythm that was slower than the rhythm of abdominal pumping movements. The same was true for the phases of rapid walking.

During chill coma experiments (see Section 2.1.3), periods of long-lasting dilations of the dorsal air sacs were also observed: during cooling of the experimental chamber to the selected chill coma level and during the subsequent rewarming of the setup to the original temperature level. These periods of long-lasting dilations were often not accompanied by rapid pumping movements of the abdomen. This was particularly 

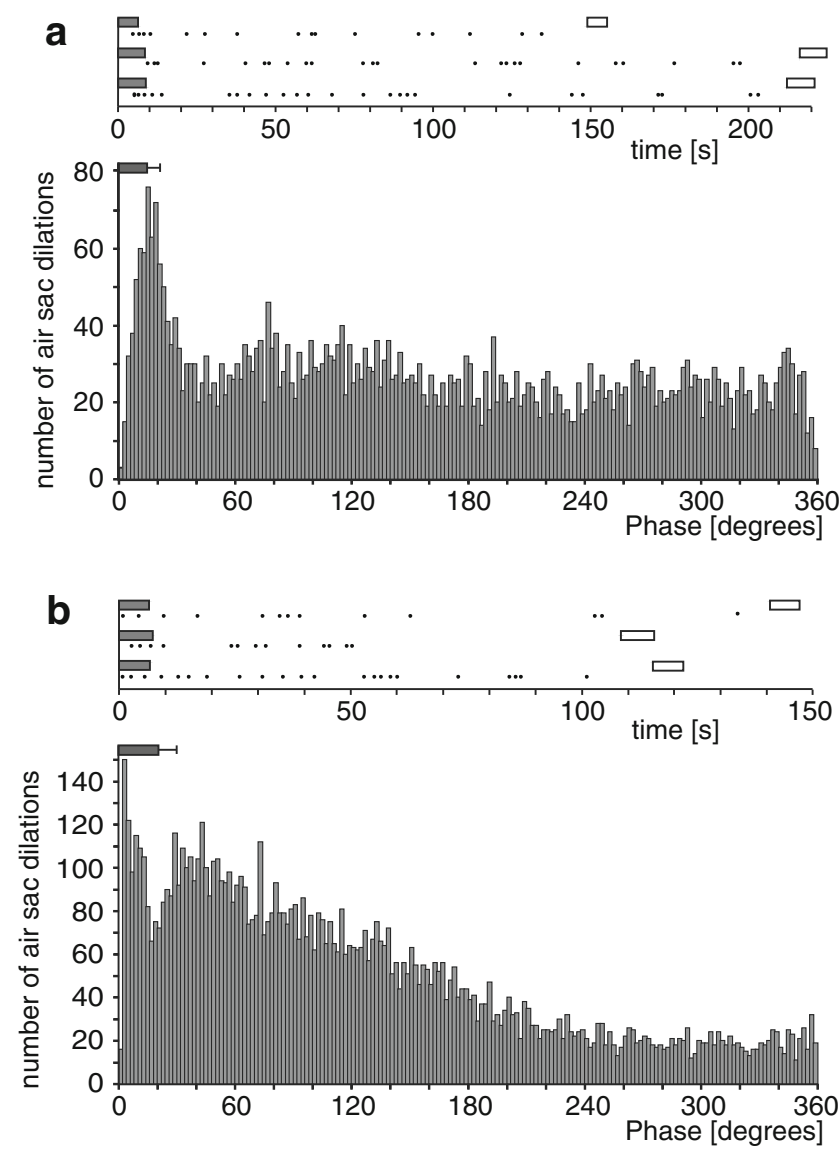

Figure 3. a, b The occurrence of dilations of dorsal air sacs during the respective ventilatory cycles (ventilatory bursts plus the following pauses in abdominal ventilation). a Bee 1, night 1 . Lights off at $2000 \mathrm{~h}$, lights on at $0645 \mathrm{~h}$. Analyzed recording period, $1950 \mathrm{~h}$ to $0730 \mathrm{~h} \mathrm{(11} \mathrm{h} 40 \mathrm{~min})$. Only dilations of the left anterior dorsal air sac of abdominal segment 5 were evaluated. Upper part of the figure: three ventilatory cycles, at $2135 \mathrm{~h}$, $0007 \mathrm{~h}$, and $0128 \mathrm{~h}$ (top to bottom). Filled rectangles: ventilatory bursts; open rectangles: the following bursts in each case, starting a new cycle. Dots mark individual air sac dilations. In order to adequately compare the events during all ventilatory cycles of the night that display considerable differences in duration, phase diagrams are shown; they contain the data from all ventilatory cycles of the analyzed recording period. The duration of each ventilatory cycle was set to a phase angle of $360^{\circ}$, abscissa. The ordinate shows the total number of air sac dilations for each $2^{\circ}$ bin, following the start of each cycle (begin of the respective ventilatory burst) during the recording period. Dark rectangle at the top of the phase diagram: average extent of all ventilatory bursts in ${ }^{\circ}$ plus standard deviation. The 304 ventilatory cycles and 4,879 air sac dilations contributed to the phase diagram. The mean duration of all ventilatory cycles was $139.5 \mathrm{~s}$ (SD $68.5 \mathrm{~s}$ ). The mean duration of the ventilatory bursts was $5.5 \mathrm{~s}$ (SD $2.8 \mathrm{~s}$ ). b Bee 2, night 1 . Lights off at $1930 \mathrm{~h}$, lights on at $0700 \mathrm{~h}$. Analyzed recording period, $1915 \mathrm{~h}$ to $0934 \mathrm{~h}(14 \mathrm{~h} 19 \mathrm{~min})$. Only dilations of the left anterior air sac of segment 6 (where the heart ends) were evaluated. Upper part of the figure: three ventilatory cycles at $2114 \mathrm{~h}$, $0114 \mathrm{~h}$, and $0421 \mathrm{~h}$ (top to bottom). Lower part of the figure: phase diagram as in a, containing all data from the recording period, 658 ventilatory cycles and 8,955 air sac dilations. Mean duration of all ventilatory cycles, $78.4 \mathrm{~s}$ (SD $31.7 \mathrm{~s})$. Mean duration of all ventilatory bursts, $4.5 \mathrm{~s}$ (SD $2.1 \mathrm{~s})$ 
evident during chill coma in bee 2 , which lasted $1 \mathrm{~h}$ and which was accompanied by miniature pumping movements of the abdomen (Kaiser 2002).

\subsubsection{The physiological basis for the dilations of the dorsal air sacs}

The fact that the volume increases of the air sacs occurred much more rapidly than the following decreases in volume points to muscles extending the air sacs (see also Section 3.2.1.3). The alary muscles of the dorsal diaphragm were considered to be likely candidates for this function (Kaiser 2001). However, this assumption is ruled out by the following observation from our study: The air sacs can be dilated independently, even in one and the same abdominal segment. For example, the posterior (elongated) left dorsal air sac in segment 5 (see Figure 2) often remained collapsed when the neighboring anterior air sac was dilated. The long-lasting dilations described in the previous section also contradict this assumption (see Section 4.1).

\subsection{Experiments combining transillumination and electrophysiology}

\subsubsection{The recorded potentials}

As stated in Section 2.2.2, we recorded summed potentials from the heart (ECG) and other muscles located in the dorsal sinus. Three types of electrical potentials could be easily discriminated by the differences in sound they produced in the loudspeaker, as well as by the differences in amplitude and time course of the signals on the oscilloscope screen. The "type 1 potentials" produced a loud and clear sound, because they had the largest amplitude (up to $2.2 \mathrm{mV}$ ) and a long duration (approximately $130 \mathrm{~ms}$ ) and appeared as discrete events without overlap. The "type 2 potentials" were much smaller in amplitude and showed considerable overlap. They produced a sharp noise in the loudspeaker. The "type 3 potentials" were also small in amplitude. Individual potentials had a duration of approximately $24 \mathrm{~ms}$ when they were negative and approximately $32 \mathrm{~ms}$ when they were positive. The "type 3 potentials" produced a characteristic dark and "fluttering" sound in the loudspeaker. The long duration of all recorded potentials shows that they originated in muscles, not nerves.

A comparison of the different sounds from the loudspeaker with the events seen simultaneously on the "transillumination video monitor" yielded a very clear-cut correspondence: The type 1 potentials were only heard when the heart contracted and were thus electrocardiogram potentials. The type 2 potentials appeared when the abdomen made ventilatory pumping movements-every shortening and every lengthening of the abdomen was accompanied by its own sharp noise, which demonstrated that antagonistic muscles of the abdomen were the source of these potentials. Finally, the type 3 potentials always and only appeared when dorsal air sacs expanded.

Electrocardiogram (ECG) potentials of regular polarity According to the literature (Snodgrass 1956; Wasserthal 1996), in the honey bee, waves of contraction run from the posterior end of the tubular heart in abdominal segment 6 anteriorly, i.e., the excitation of the heart muscle must start in segment 6 . This agrees well with the polarity of the components of the ECG potentials we observed in most cases. In order to understand the meaning of the form of the individual potentials in this paper, the following facts are relevant: (1) In extracellular recordings from a muscle, the electrode which is close to the excited part of the muscle becomes transiently negative with respect to the electrode, which is close to a non-excited part of the muscle. (2) Segment 6 of the abdomen was connected to the negative input of the differential amplifier and segment 5 to its positive input (see Section 2.2.2). Consequently, an excitation starting in segment 6 must lead to a potential that starts with a positive (upward) deflection. 
This is, indeed, the case in the majority of cases (see Figure 4). We termed these ECG potentials thus "potentials of regular polarity."

However, Figure 4 also shows one potential of reversed polarity (arrow), starting with a negative deflection, thus indicating that the excitation started in the portion of the heart situated in segment 5. The position of this ECG potential of reversed polarity on the time axis suggests the following interpretation: This excitation in segment 5 appeared earlier than the next regular wave of excitation elicited in segment 6 would have reached segment 5 . Thus, the heart section in segment 5 was still refractory, due to this extra systole, and the next regular excitation originating in segment 6 could not become effective in segment 5 .

The following conclusion must be drawn from these findings: The regular ECG potentials starting with a positive deflection reflect forward pumping of the heart (from caudal to rostral), whereas reversed ECG potentials reflect backward pumping (from rostral to caudal). Thus, heartbeat reversal does, indeed, exist in the honey bee - at least in that part of the heart from which we recorded.

ECG potentials of reversed polarity Potentials of reversed polarity appeared in all four experiments, either as single events, as in Figure 4, or in groups of two or three. In addition, in the three chill coma experiments that were performed with

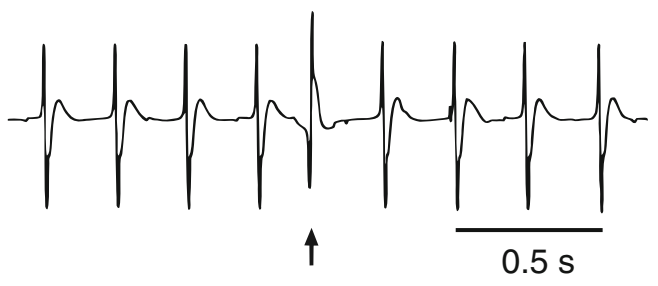

Figure 4. Section of an electrocardiogram recording of a worker honey bee. A group of regular cardiac potentials that traversed the cardiac tube from caudal to rostral (forward pumping of the heart) contained a single reversed potential (arrow) that moved in the opposite direction (backward pumping). Scale bar, $0.5 \mathrm{~s}$ three of the four animals, groups of reversed potentials alternated with groups of regular potentials when the animals were cooled down to the chill coma temperature and also when they later were re-warmed to the original ambient temperature (see Section 2.1.3). During these phases, continuous pumping movements of the abdomen appeared, which are a reliable sign of heat production with the thoracic muscles (see Kaiser 1988). During the chill coma phases (stable ambient temperature of $5.8-5.9^{\circ} \mathrm{C}$ ), single potentials starting with a positive deflection alternated with single reversed potentials.

Potentials from unidentified muscles The statement that the expansions of the air sacs are always accompanied by muscle potentials that produce a characteristic, unmistakable sound in the loudspeaker (type 3 potentials) is substantiated by the following analysis of a video tape that contained the transillumination recording and, in addition, the sound produced by the simultaneously occurring electrophysiological signals on its sound track (see Section 2.2.2 and see and hear the videos $\mathrm{S} 1$ and $\mathrm{S} 2$ contained in the supplementary material).

Five separate video clips (total duration, $84 \mathrm{~min}$ ) from animal no. 2, studied in September during sleep and rest, were analyzed. A total of 1,025 dilations of the left dorsal air sacs occurred either in the abdominal segments 5 or 6 , or simultaneously in segments 5 and 6 . In all cases, type 3 potentials were associated with dilations of air sacs. There was no dilation that was not accompanied by these potentials. The (slower) collapse of the air sacs following each dilation was not accompanied by electrical potentials and thus is probably due to elastic components in the walls of the air sacs. Since we do not know which muscles expand the air sacs, we call the type 3 potentials "potentials from unidentified muscles."

The clock times and results of the five video clips were 1215-1230 h, 100 dilations; 21272148 h, 171 dilations; 0736-0817 h, 551 dilations; 1011-1013 h, 60 dilations; and 1014-1019 h, 133 dilations. The nightly dark 
phase had started at $2015 \mathrm{~h}$ and ended at $0645 \mathrm{~h}$.

An essential indication related to the nature of the "unidentified muscles" came from the chill coma experiments that were combined with transillumination and electrophysiology: During the cooling phases as well as during the rewarming of animal and experimental setup following chill coma, phases of continuous air sac expansion (no air sac "pulsations" for, e.g., $40 \mathrm{~min}$ ) that were accompanied by continuous activity of the unidentified muscles appeared. Since their potentials were not superimposed on the activity of muscles responsible for abdominal ventilatory movements (which was absent), the potentials of the unidentified muscles could be easily recognized in the sound recording. The significance of this finding will be discussed in Section 4.1; see also Section 3.1.4).

\subsection{Electrophysiology and behavior without transillumination}

\subsubsection{Potentials from unidentified muscles}

As stated in Section 3.1.2, the expansions of the dorsal air sacs were associated with an acceleration of heartbeat. In Section 3.2.1.3, it was shown that the expansions of the air sacs were caused by "unidentified muscles." Figure 5 demonstrates an example of a simultaneous recording of potentials from these unidentified muscles and ECG potentials. The appearance of potentials from unidentified muscles (numbered 1-3) was associated with a temporary increase in the frequency of the regular ECG potentials starting with a positive deflection (forward pumping of the heart). Figure 6 shows a quantitative evaluation of the data from Figure 5. There was a threefold increase of heartbeat rate, which slowly declined after the potentials from unidentified muscles (marked by the bars below) had ceased.

\subsubsection{Electrocardiogram potentials}

In this experimental series, ECG potentials of reversed polarity also appeared, either as single events or in groups of a few pulses. In one of the four experiments (animal 4, wings trimmed but all legs left intact), large groups of reversed ECG potentials (backward pumping of the heart) that alternated with groups of regular ECG potentials occurred. This phenomenon was already seen in the chill coma experiments described in Section 3.2.1.2. However, here, the phenomenon of heartbeat reversal occurred spontaneously-during both experimental nights studied in this animal as well as during rest in the morning after lights-on. Figure 7 shows a recording that was made in the morning following night 1 , while the bee was still resting. Ventilation was still discontinuous (upper record: abdominal length), as it had been throughout the night. There was no fixed relationship between the bursts of ventilatory movements and the appearance of heartbeat reversals (lower record). The ECG potentials of reversed polarity (backward pumping, marked by horizontal bars below) were of larger amplitude than the regular ECG potentials (forward pumping). A very important feature, evident in this figure, is the following: The signals from unidentified muscles (arrow) appeared when the abdomen showed no externally visible motion. Additionally, the occurrence of potentials from unidentified muscles was accompanied by an abrupt transformation of ECG potentials with reversed polarity into ECG potentials with regular polarity, whose frequency increased transiently.

General features during the appearance of heartbeat reversals in both nights were: The bee did not perform body movements, except for rhythmical length changes of the abdomen during discontinuous resting ventilation. The antennae also displayed the usual degree of nightly immobility. In addition, during rest in the morning after lights-on, self-grooming that was accompanied by a series of ECG potentials of reversed polarity of high frequency (mean value, $6.9 \mathrm{~Hz}$ ) was observed. When the bee drank diluted honey, offered by a cannula, a group of ECG potentials with reversed polarity 


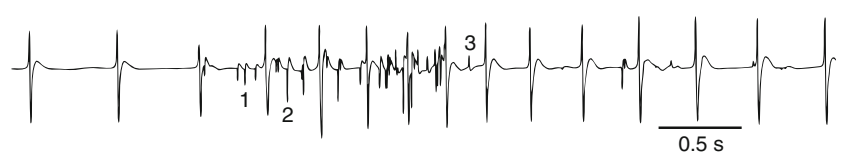

Figure 5. Section of an electrophysiological recording containing the ECG and-superimposed-potentials from "unidentified muscles." In the presence of signals from "unidentified muscles," there was a temporary increase in the frequency of the regular potentials associated with forward pumping of the heart. The signals from "unidentified muscles" differ in their form; for those numbered 1 and 2, the largest component of the potential was negative, for number 3 it was positive. Animal 4, night no. 2; $0617 \mathrm{~h}$ (4 min after lights-on). Scale bar, $0.5 \mathrm{~s}$

also occurred that had an even higher frequency $(8 \mathrm{~Hz})$, whereas, during the following period of active warming-up (characterized by persistent abdominal ventilatory movements), only regular ECG potentials (forward pumping of the heart) of high amplitude that had a frequency of $5.7 \mathrm{~Hz}$ appeared.

\section{DISCUSSION}

\subsection{Active oxygen supply of the heart during sleep and rest}

A key result of this paper is the discovery of an active ventilatory mechanism providing gaseous exchange for the muscle cells of the heart during sleep and rest in honey bees. A preliminary report of our findings has already appeared (Kaiser 2001).

The dorsal air sacs of the abdomen were found to undergo volume changes (see Figure 2 and also Videos S1 and S2 in the supplementary material) that also occur when the abdomen is motionless and thus not engaged in ventilatory pumping movements (Figure 3). These air sacs are connected with the large lateral air sacs via tracheal tubes and thus to the outside world. The heart seems to be the main organ receiving tracheal supply from the dorsal air sacs via fine tracheal arborizations (Figure 2 and Snodgrass 1956). Volume changes of the dorsal air sacs must therefore result in an active exchange of respiratory gases to and from the heart muscle cells during a phase when formerly only diffusion of gases was believed to occur. This is of particular importance since, during discontinuous resting ventilation, the ventilatory pauses are much longer than the ventilatory bursts. In bee 1 , the ventilatory cycles (duration of burst plus pause) were, on average, 24 times as long as the bursts. In bee 2, the factor was 16.6 (see caption of Figure 3 for details).

As shown in Section 3.2.1.3, the increase in volume of the air sacs is the active process, not the decrease in volume. The latter probably is due to elastic components in the walls of the air sacs. Originally, the alary muscles of the dorsal

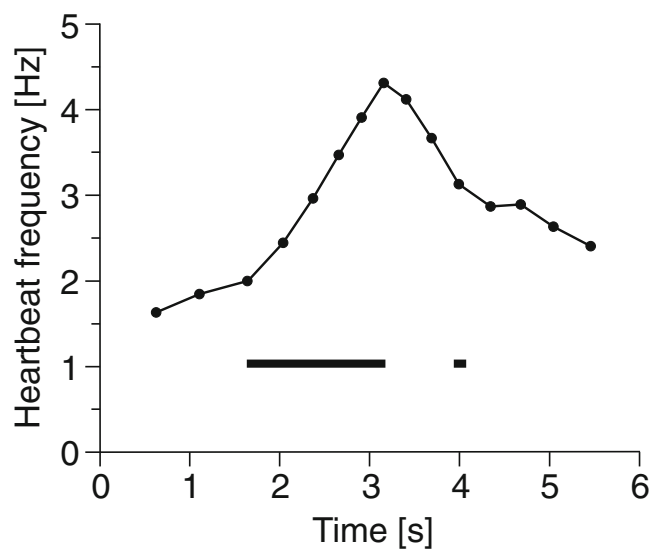

Figure 6. Heartbeat frequency as a function of time (quantitative representation of the increase in frequency of the regular potentials in Figure 5). Each frequency value was calculated from the inverse of the respective potential interval. The horizontal bars indicate the presence of signals from "unidentified muscles" 


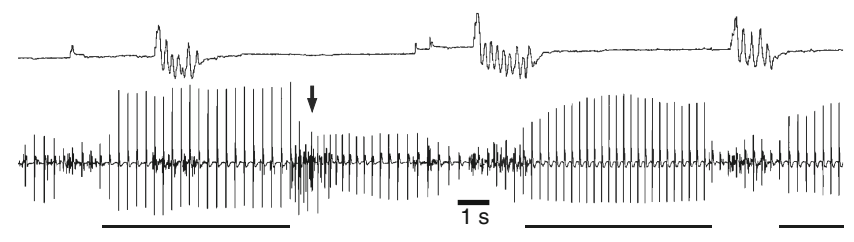

Figure 7. Recordings of the abdominal length (upper trace) and a section of an electrophysiological recording containing electrocardiogram (ECG) potentials and potentials from muscles in the dorsal sinus (lower trace). The recording of the abdominal length shows the individual ventilatory movements of the ventilatory bursts that occurred during discontinuous resting ventilation. Upward displacement of the trace: extension of the abdomen. Lower trace: Groups of reversed ECG potentials (indicated by horizontal bars) alternated with groups of regular ECG potentials of lower amplitude. Arrow: Presence of signals from "unidentified muscles." Signals from muscles producing length

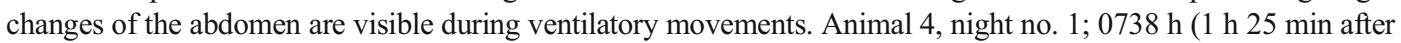
lights-on). Scale bar under the recording of abdominal position, $1.0 \mathrm{~s}$

diaphragm were assumed to be the motor for the dilations of the air sacs (Kaiser 2001). The fact that, under certain conditions, long-lasting dilations of the air sacs occur (see Section 3.1.3) that are accompanied by long-lasting activity of the muscles whose activity is also associated with the transient dilations (see Section 3.2.1.3) argues strongly against the alary muscles as the motor for the observed dilations.

The following hypothesis was presented by Kaiser (2001): Contractions of the alary muscles in the dorsal diaphragm lead to a volume increase of the pericardial sinus above the diaphragm, whereby hemolymph of the perivisceral sinus is sucked into the pericardial sinus. A transient pressure reduction in this hemolymph space at the beginning of the alary muscles' contraction was thought to be the cause of the transient volume increases of the dorsal air sacs lying in the pericardial sinus. However, this mechanism would only explain the transient dilations of the air sacs and not the observed long-lasting dilations, since, during longer-lasting contractions of the dorsal diaphragm, the dorsal sinus would be filled with hemolymph. This would lead to a shrinkage of the air sacs lying in this hemolymph space.

Because we do not know which muscles cause the dilations of the dorsal air sacs, we call them "unidentified muscles" in this paper.
During discontinuous resting ventilation of the abdomen, which is a characteristic feature of sleep and rest in honey bees, short bursts of ventilatory pumping movements are followed by longer pauses, during which these movements are absent (Figure 7). The bursts of pumping movements are, on average, associated with an increase in the frequency of the volume changes of the dorsal air sacs (Figure 3). However, the abdominal pumping movements are not the cause of the volume changes of the dorsal air sacs, since there is no synchrony between these pumping movements and the volume changes of the air sacs. The correlation between the appearance of abdominal ventilatory bursts and the increase in the rate of air sac dilations must indicate an improvement of the gaseous exchange for the heart muscles because, during the bursts, the large lateral air sacs (to which the dorsal air sacs are connected) are ventilated. The active oxygen transport to the heart then continues throughout the pauses in abdominal ventilation: Volume changes of the dorsal air sacs occur repeatedly, and their rate reaches a plateau-like level (Figure 3).

A further important result is the observed relationship between volume changes of the dorsal air sacs and heartbeat frequency: The occurrence of air sac dilations is always associated with an increase in heart rate (see 
Sections 3.1.2, 3.2.1.3, and 3.3.1, as well as Figures 5 and 6). In addition, during pauses in heart activity (during sleep and rest), the heart starts beating when air sac dilations occur (or vice versa). Thus, the heart is always supplied with oxygen when it needs it.

\subsection{Electrocardiogram potentials with reversed polarity}

The existence of potentials with reversed polarity was first reported by Kaiser and Weber (1998). The surprising fact that such potentials were not discovered during the electrophysiological study of honey bees by Schwab et al. (1991) is easily explained: Schwab et al. positioned their recording electrodes in one and the same abdominal segment (segment 5), whereas we placed them in different segments (5 and 6), behind each other, next to the tubular heart (which ends in segment 6). Also, Schwab et al. used impedance conversion, whereas we measured potential differences with a differential amplifier.

Heart beat reversal is known to exist in several insect orders (for literature see Wasserthal 1996). The fact that Wasserthal and his group (see Wasserthal 1996, for review) did not detect episodes of backward beating in their physiological study of the honey bee heart, and thus heartbeat reversal might be explained by the following: (1) we made long-lasting ECG recordings (days), (2) our bees were well-accustomed to the experimental situation; they even showed sleep which is a behavior easily disturbed if the animals feel uncomfortable. Sleep was, in our experiments, continuously monitored by recording the animal's behavior on the treadwheel simultaneously with the continuous electrophysiological recording (see Figure 1).

Heartbeat reversal described in our paper is definitely not a pathological phenomenon. Periodic heartbeat reversal (see Figure 7) was found during long-lasting recording in four bees. Three of the bees were completely intact, whereas in one animal the wings were trimmed. Nevertheless, the behavior of this bee also appeared to be totally normal.
Our study only demonstrates heartbeat reversal in the rear portion of the tubular heart, where our electrodes were positioned. Future studies have to clarify whether the rostral continuation of the honey bee's heart, the "aorta," also participates in heartbeat reversal. This is the case in certain insects at rest (see Wasserthal 1996). A characteristic feature of heartbeat reversal in these insects is the strict coordination of the ventilatory bursts of the abdomen with the appearance of phases of forward beating.

In honey bees, we did not find such coordination between bursts of ventilatory movements and the direction of heartbeat - neither with forward beating, nor backward beating (see Figure 7). This favors the interpretation that heartbeat reversal in honey bees has a function which is different from that in the insects studied by Wasserthal (1996), where the periodic change of the direction of heartbeat serves a ventilatory function: The air sacs in the rostral part of the body are compressed, and the ones in the caudal section are inflated when the heart beats forward and vice versa during backward beating.

A plausible interpretation of heartbeat reversal described here in the honey bee is the following: Heartbeat reversal in sleeping and resting bees is confined to the abdomen and thus to the heart in the strict sense. Such a "short cut circulation within the abdomen" was proposed for lepidopteran pupae by Wasserthal (1996). What could be the function of such a circulation mode in an adult sleeping or resting honey bee? We suggest that it might facilitate the uptake of nutrients by the hemolymph, which then has a more intense contact with the abdominal organs.

\section{ACKNOWLEDGMENTS}

The constructive criticism provided by the referees is gratefully acknowledged. One of us (W.K.) thanks the Max-Planck-Institut für Verhaltensphysiologie (now Max-Planck-Institut für Ornithologie) for its hospitality during several stays as guest researcher. $\mathrm{He}$ is also grateful to Peter Dancker (Heidelberg) for numerous valuable suggestions to the manuscript. The experiments on animals reported here have been registered with the appropriate authority. 
Apport en oxygène du coeur et potentiels de l'électrocardiogramme avec une polarité inversée chez les abeilles en état de sommeil ou de repos

\section{Abeille / sommeil / fonction du coeur / apport en oxygène / électrocardiogramme}

\section{Die Sauerstoffversorgung des Herzens und Elektrokardiogrammpotenziale mit umgekehrter Polarität in schlafenden und ruhenden Honigbienen}

\section{Honigbiene / Schlaf / Herzfunktion / Sauerstoffversorgung / Elektrokardiogramm}

\section{REFERENCES}

Beyaert, L., Greggers, U., Menzel, R. (2012) Honeybees consolidate navigation memory during sleep. J. Exp. Biol. 215, 3981-3988

Dement, W.C. (2011) History of sleep physiology and medicine. In: Kryger, M.H., Roth, T., Dement, W.C. (eds.) Principles and practice of sleep medicine, 5th edn, pp. 3-15. Elsevier/Saunders, St Louis

Eban-Rothschild, A., Bloch, G. (2012) Circadian rhythms and sleep in honey bees. In: Galizia, C.G., Eisenhardt, D., Giurfa, M. (eds.) Honeybee neurobiology and behavior: a tribute to Randolf Menzel, pp. 31-45. Springer, Dordrecht-Heidelberg-LondonNew York

Kaiser, W. (1988) Busy bees need rest, too: behavioural and electromyographical sleep signs in honeybees. J. Comp. Physiol. A 163, 565-584

Kaiser, W. (1995) Rest at night in some solitary bees-a comparison with the sleep-like state of honey bees. Apidologie 26, 213-230

Kaiser, W. (2001) A "new" function of the dorsal diaphragm of the honey bee. In: Elsner, N., Kreutzberg, G.W. (eds.) Göttingen neurobiology report 2001, vol. II, p. 332. Georg Thieme Verlag, Stuttgart-New York
Kaiser, W. (2002) Honey bee sleep is different from chill coma: behavioural and electrophysiological recordings in forager honey bees. J. Sleep Res. 11(Suppl. 1), 115

Kaiser, W., Faltin, T., Bayer, G. (2002) Sleep in a temperature gradient: behavioural recordings from forager honey bees. J. Sleep Res. 11(Suppl. 1), 115-116

Kaiser, W., Weber, T., Otto, D. (1996) Vegetative physiology at night in honey bees. In: Elsner, N., Schnitzler, H.-U. (eds.) Göttingen neurobiology report 1996, vol. II, p. 140. Georg Thieme Verlag, Stuttgart-New York

Kaiser, W., Weber, T. (1998) Heartbeat reversal in honey bees. In: Elsner, N., Wehner, R. (eds.) Göttingen neurobiology report 1998, vol. II, p. 251, p. Göttingen neurobiology report 1998, vol. II, p. 251. Georg Thieme Verlag, Stuttgart-New York

Klein, B.A., Klein, A., Wray, M.K., Mueller, U.G., Seeley, T.D. (2010) Sleep deprivation impairs precision of waggle dance signaling in honey bees. Proc. Natl. Acad. Sci. 107, 22705-22709

Klein, B.A., Seeley, T.D. (2011) Work or sleep? Honeybee foragers opportunistically nap during the day when forage is not available. Anim. Behav. 82, $77-83$

Peigneux, P., Smith, C. (2011) Memory processing in relation to sleep. In: Kryger, M.H., Roth, D., Dement, W.C. (eds.) Principles and practice of sleep medicine, 5th edn, pp. 335-347. Elsevier/Saunders, St Louis

Sauer, S., Kinkelin, M., Herrmann, E., Kaiser, W. (2003) The dynamics of sleep-like behaviour in honey bees. J. Comp. Physiol. A 189, 599-607

Sauer, S., Herrmann, E., Kaiser, W. (2004) Sleep deprivation in honey bees. J. Sleep Res. 13, 145-152

Schmolz, E., Hoffmeister, D., Lamprecht, I. (2002) Calorimetric investigations on metabolic rates and thermoregulation of sleeping honeybees (Apis mellifera carnica). Thermochim. Acta 382, 221-227

Schwab, E.R., Chilson, R.A., Eddleman, C.D. (1991) Heartbeat rate modulation mediated by the ventral nerve cord in the honey bee, Apis mellifera. J. Comp. Physiol. B 161, 602-610

Snodgrass, R.E. (1956) Anatomy of the honey bee. Comstock Publishing Associates, Ithaca-London

Wasserthal, L.T. (1996) Interaction of circulation and tracheal ventilation in holometabolous insects. Adv. Insect Physiol. 26, 297-351 